\title{
Cytotoxic evaluation of substituted azetopyrroloazepinones
}

\author{
Roberto Martínez, ${ }^{1}$ * Gustavo Ávila-Zárraga, ${ }^{2}$ Ma. Teresa Ramírez ${ }^{1}$ and Araceli Pérez ${ }^{1}$ \\ Instituto de Química, ${ }^{1}$ Facultad de Química, ${ }^{2}$ Universidad Nacional Autónoma de México. \\ Circuito Exterior, Ciudad Universitaria, 04510 Coyoacán, México, D. F. \\ E-mail: robmar@servidor.unam.mx
}

(received 25 Jul 03; accepted 09 Oct 03; published on the web 17 Oct 03)

\begin{abstract}
Azetopyrroloazepinones 1a-g were synthesized in six steps from 5,5-dimethyl-1,3cyclohexanedione as starting material in good yields. These compounds were tested on five tumoral cell lines with the aim of elucidating the relationship between the substituents attached to the 3-phenyl ring and their cytotoxic activity. The results are inconclusive in regard to the relationship between the physicochemical properties of the substituents on the 3-phenyl group of azeto-pyrroloazepinones 1a-g and the inhibition of the growth in tumor cell lines. However, the results show a clear link between the presence of halogens on position 3- or 4- and the cytotoxicity of these compounds.
\end{abstract}

Keywords: Azeto-pyrroloazepinones, cancer cell lines, cytotoxic activity

\section{Introduction}

Cancer is one of the main causes of death in the world despite considerable progress in the understanding of its biology and pharmacology. The traditional therapeutic strategies for the treatment of this disease are surgery, radiotherapy, immunotherapy and chemotherapy. For the time being, $50 \%$ of the patients diagnosed with cancer are cured either through one of these methods or by a combination of them. For some types of disseminated cancers, chemotherapy is the only effective therapy because it distributes anticancer drugs through the circulatory system. ${ }^{1}$ We are currently engaged in a program aimed at synthesizing novel heterocyclic compounds that inhibit the growth of cancer cells. ${ }^{2}$ One of these compounds is azetopyrroloazepinone 1a, which showed in vitro cytotoxic activity against PC-3 (prostate) and U251 (CNS) cancer cells lines. ${ }^{3}$ The mechanism of action of antiproliferative activity of compound 1a is still unknown, therefore, further exploration of the azetopyrroloazepinone pharmacophore is advisable. The aim of the present study was both to explore the role of the substituents attached to the 3-phenyl ring of compounds 1a-g on the inhibition of cancer cell growth, and to identify an optimal candidate 
among currently available compounds. It was also intended to ascertain potential directions for synthetic lead-optimization studies. ${ }^{4}$

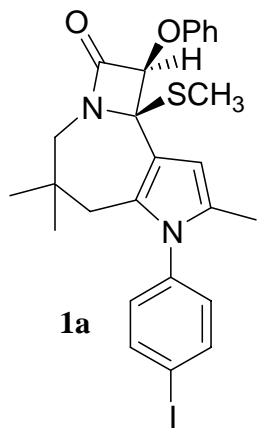

Compounds 1b-e were prepared in order to study the tendency of halogens to present activity. In addition, compounds $\mathbf{1 f}\left(\mathrm{R}=\mathrm{CH}_{3}\right)$ and $\mathbf{1 g}\left(\mathrm{R}=\mathrm{OCH}_{3}\right)$ were included in the study to investigate the electron donating effects of $-\mathrm{CH}_{3}$ and $-\mathrm{OCH}_{3}$ groups.

\section{Results and Discussion}

\section{Chemistry}

For this study, we synthesized a set of derivatives as previously described (Scheme 1). ${ }^{5}$

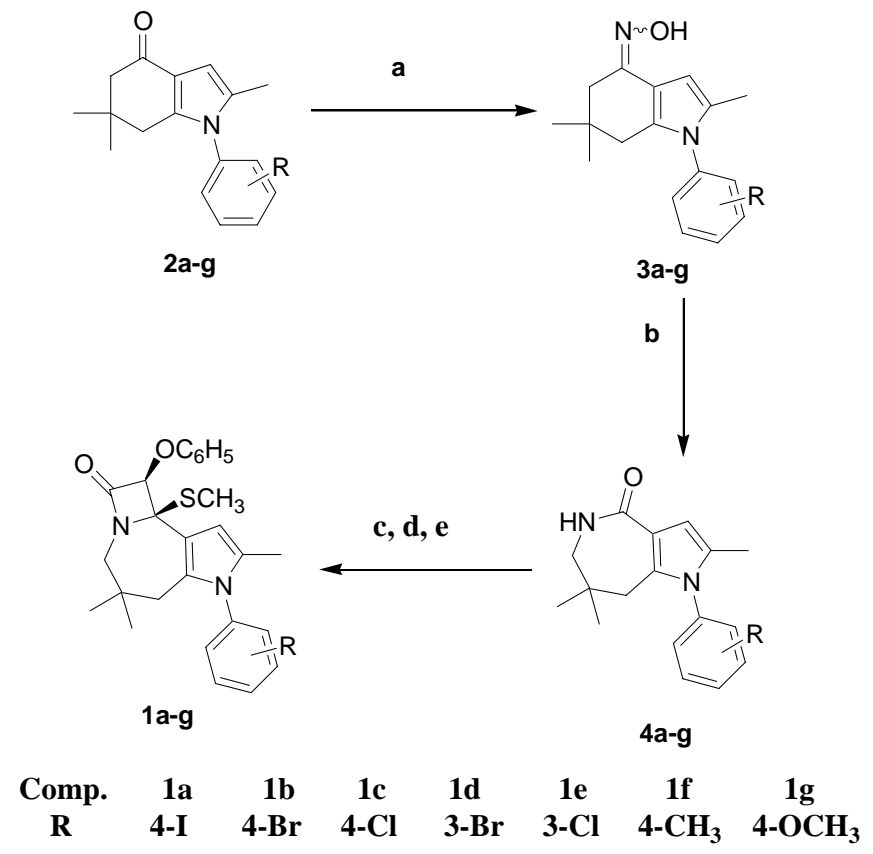


Scheme 1. (a) $\mathrm{NH}_{2} \mathrm{OH} \cdot \mathrm{HCl}$, AcONa, $\mathrm{H}_{2} \mathrm{O}$, EtOH, $2 \mathrm{~h}$, reflux; (b) PPA, 90-120 ${ }^{\circ} \mathrm{C}, 3 \mathrm{~h}$; (c) Lawesson's reagent, toluene, $1-2 \mathrm{~h}$; (d) (i) $\mathrm{CH}_{3} \mathrm{I}, \mathrm{CH}_{2} \mathrm{Cl}_{2}$, rt, $1 \mathrm{~h}$; (ii) $\mathrm{NaHCO}_{3}$ (aq) (e) $\mathrm{PhOCH}_{2} \mathrm{COCl}, \mathrm{NEt}_{3}, \mathrm{C}_{6} \mathrm{H}_{6}$, reflux, $8 \mathrm{~h}$.

This synthetic approach starts from the Beckmann rearrangement of tetrahydro-4-indoloneoximes 3a-g, easily prepared from tetrahydroindol-4-ones 2a-g, to the corresponding azepinones 4a-g, which are transformed to methylsulfanyl-hexahydropyrrolo-azepines. These imines react with phenoxyacetyl chloride (Staundinger reaction) to give the 3-aryl-2, 5, 5-trimethyl-9a(methylsulfanyl)-9-phenoxy-4, 5, 6, 8, 9, 9a-hexahydro-3H-azeto[1,2-] pyrrolo[3,2-c]azepin-8ones 1a-g. While the Beckmann reaction occurred in a regioselective way, the Staudinger reaction proceeded with high stereoselectivity. All the compounds were purified either by recrystallization in hexane or by silica gel column chromatography.

\section{Antiproliferative activity}

Azeto-pyrroloazepinones 1a-g were evaluated in vitro for their ability to inhibit the growth of PC-3 prostate, U251 central nervous system, K652 leukemia, HCT-15 colon and MFC7 breast cancer cells. The percentage of inhibition of the growth of the five tumoral cell lines after treatment with each compound at a concentration of $100 \mu \mathrm{M}$ is given in Table 1 and the $\mathrm{IC}_{50}$ values $(\mu \mathrm{M})$ on Table 2 .

Table 1. \% Inhibition of growth of compounds 1a-g to the five cancer cell lines

\begin{tabular}{|ccccccc|}
\hline Compound & $\mathrm{R}$ & $\begin{array}{c}\text { PC-3 } \\
\text { (prostate) }\end{array}$ & $\begin{array}{c}\text { U-251 } \\
\text { (CNS) }\end{array}$ & $\begin{array}{c}\text { K562 } \\
\text { (leukemia) }\end{array}$ & $\begin{array}{c}\text { HCT-15 } \\
\text { (colon) }\end{array}$ & $\begin{array}{c}\text { MCF-7 } \\
\text { (breast) }\end{array}$ \\
\hline 1a & $4-\mathrm{I}$ & 64.71 & 68.04 & 37.90 & 35.74 & 46.10 \\
\hline 1b & $4-\mathrm{Br}$ & 90.43 & 99.98 & 40.59 & -29.33 & 73.20 \\
\hline 1c & $4-\mathrm{Cl}$ & 78.9 & 49.44 & 77.64 & -45.59 & 45.59 \\
\hline $\mathbf{1 d}$ & $3-\mathrm{Br}$ & 61.88 & 33.29 & 65.76 & 44.67 & 57.03 \\
\hline $\mathbf{1 e}$ & $3-\mathrm{Cl}$ & 76.07 & 43.73 & 59.34 & -169.0 & 21.78 \\
\hline $\mathbf{1 f}$ & $4-\mathrm{CH} 3$ & 54.58 & 18.33 & 46.05 & 40.10 & 38.51 \\
\hline $\mathbf{1 g}$ & $4-\mathrm{OCH} 3$ & 68.81 & 34.06 & 64.77 & 52.71 & 55.39 \\
\hline
\end{tabular}

Compounds 1a-g were examined to analyze the importance of the relative position of the substituents attached to the 3-phenyl ring of these compounds and their cytotoxic activity (Table 2). The inhibition resulted by 1a lead to the conclusion that electronegativity and/or size of halogens could be implicated in the cytotoxic activity. To test this idea, compounds $\mathbf{1 b}$ and $\mathbf{1 c}$ were synthesized. The series of 4-halogen derivatives showed that activity followed the order $\mathbf{1 c}(\mathrm{Cl})>\mathbf{1} \mathbf{b}(\mathrm{Br})>\mathbf{1 a}(\mathrm{I})$. To complete the series of halogen compounds, $\mathbf{1 d}(3-\mathrm{Br})$ and $\mathbf{1 e}(3-\mathrm{Cl})$ were obtained and they showed a greater activity than the 4-halogens derivatives. 
These results indicated an apparent relationship between both the electronegativity and the size of the halogen versus the cytotoxic activity of the 4-halogen derivatives. However, this statement is not clear in the case of 3-halogen derivatives. On other hand, compound $\mathbf{1 f}\left(4-\mathrm{CH}_{3}\right)$ was the most selective displaying a relatively good inhibition in the PC-3 cell line. In contrast, compound $\mathbf{1 g}\left(4-\mathrm{OCH}_{3}\right)$ was the less selective. An analysis of the cytotoxic activity by cell line type indicated that all compounds 1a-g are active on the PC-3 cell line, derivative 1d being four times more active than the leading compound 1a. In contrast, on U-251 cell line, all compounds $\mathbf{1 b}$-g tested showed a complete lack of activity. Compound $\mathbf{1 g}\left(4-\mathrm{OCH}_{3}\right)$ is the only one that induces cytotoxic activity on the HCT-15 cell line. In the case of K-562 and MFC-7 cell lines the compounds 1a-g did not show any tendency.

Table 2. The IC 50 values $(\mu \mathrm{M})$ of compounds 1a-g to the five cancer cell lines

\begin{tabular}{|ccccccc|}
\hline Compound & $\mathrm{R}$ & $\begin{array}{c}\text { PC-3 } \\
\text { (prostate) }\end{array}$ & $\begin{array}{c}\text { U-251 } \\
\text { (CNS) }\end{array}$ & $\begin{array}{c}\text { K-562 } \\
\text { (leukemia) }\end{array}$ & $\begin{array}{c}\text { HCT-15 } \\
\text { (colon) }\end{array}$ & $\begin{array}{c}\text { MCF-7 } \\
\text { (breast) }\end{array}$ \\
\hline 1a & $4-\mathrm{I}$ & $87.0 \pm 8.6$ & $40.0 \pm 3.6$ & $>100$ & $>100$ & $>100$ \\
\hline 1b & $4-\mathrm{Br}$ & $69.58 \pm 1.1$ & $>100$ & $>100$ & $>100$ & $35.29 \pm 1.1$ \\
\hline 1c & $4-\mathrm{Cl}$ & $45.74 \pm 1.1$ & $>100$ & $50.85 \pm 1.1$ & $>100$ & $>100$ \\
\hline 1d & $3-\mathrm{Br}$ & $20.79 \pm 1.0$ & $>100$ & $20.46 \pm 1.4$ & $>100$ & $51.64 \pm 1.2$ \\
\hline 1e & $3-\mathrm{Cl}$ & $37.46 \pm 1.5$ & $>100$ & $20.70 \pm 1.8$ & $>100$ & $>100$ \\
\hline $\mathbf{1 f}$ & $4-\mathrm{CH} 3$ & $26.89 \pm 1.1$ & $>100$ & $>100$ & $>100$ & $>100$ \\
\hline 1g & $4-\mathrm{OCH} 3$ & $29.44 \pm 1.0$ & $>100$ & $21.13 \pm 1.3$ & $50.93 \pm 1.1$ & $64.46 \pm 1.0$ \\
\hline Doxorubicine & & $0.32 \pm 0.02$ & $0.09 \pm 0.02$ & $0.28 \pm 0.01$ & $0.23 \pm 0.01$ & $0.4 \pm 0.01$ \\
\hline
\end{tabular}

\section{Conclusions}

The data presented here are inconclusive in regard to the relationship between electronegativity and size of the halogens substituents on the 3-phenyl group of azeto-pyrroloazepinones 1a-g and the inhibition of the growth in tumor cell lines. However, the results show a clear link between the presence of halogens on position 3- or 4- and the cytotoxicity of these compounds. Likewise, this study reveals the interesting finding that the compounds that presented cytotoxic activity were primarily those containing halogens mainly on position 3- or a $\mathrm{CH}_{3^{-}} /-\mathrm{OCH}_{3}$ group on position.4-.

\section{Experimental Section}

General Procedures. All reactions were performed in oven-dried glassware under a positive pressure of nitrogen. Reaction mixtures and chromatography fractions were concentrated by 
using a rotary evaporator (ca. $20{ }^{\circ} \mathrm{C} / 20$ Torr). For column chromatography, the Merck silica gel 60 F254 was employed. Commercial grade reagents were used without further purification except when indicated. Benzene and toluene were distilled from a sodium/ benzophenone mixture immediately prior to use, and $\mathrm{CH}_{2} \mathrm{Cl}_{2}$ from $\mathrm{P}_{2} \mathrm{O}_{5}$.

1-(R-Phenyl)-2,6,6-trimethyl-4,5,6,7-tetrahydroindol-4-ones (2a-g). Compounds 2a-g have been prepared following a reported procedure: $\mathbf{2 c}$, mp $171-173{ }^{\circ} \mathrm{C}$ (lit. ${ }^{6} 170-172{ }^{\circ} \mathrm{C}$ ); $2 \mathbf{2 f}$, mp $170-$ $171{ }^{\circ} \mathrm{C}$ (lit. ${ }^{6} 170-172{ }^{\circ} \mathrm{C}$ ); $2 \mathrm{~g}$, mp $143-145{ }^{\circ} \mathrm{C}$ (lit. ${ }^{6} 142-144^{\circ} \mathrm{C}$ ). The spectral data where in agreement with the reported data. ${ }^{6}$ Spectral data for the unknown tetrahydroindol-4-ones $\mathbf{2 a}, \mathbf{2 b}$, 2d and $2 \mathbf{e}$ are described below.

1-(4-Iodophenyl)-2,6,6-trimethyl-4,5,6,7-tetrahydroindol-4-one (2a). mp 172-173 ${ }^{\circ} \mathrm{C}$; IR $\left(\mathrm{CHCl}_{3}\right)$ $v_{\max }\left(\mathrm{cm}^{-1}\right) 1648 ;{ }^{1} \mathrm{H}-\mathrm{NMR}\left(\mathrm{CDCl}_{3}, 200 \mathrm{MHz}\right) \delta 1.06\left(\mathrm{H}-9,9^{\prime}\right), 2.04(\mathrm{H}-8), 2.35(\mathrm{H}-5), 2.38(\mathrm{H}-7)$, 6.36(H-3), 6.9-7.87(Ar-H); MS (EI) m/z (relative intensity) 379 ( $\left.\mathrm{M}^{+}, 100\right)$; HRMS (EI) Calcd for $\mathrm{C}_{17} \mathrm{H}_{18} \mathrm{NOI} 379.0433$, Found 379.0454.

1-(4-Bromophenyl)-2,6,6-trimethyl-4,5,6,7-tetrahydroindol-4-one (2b). mp 171-173 ${ }^{\circ} \mathrm{C}$; IR $\left(\mathrm{CHCl}_{3}\right)$ $v_{\max }\left(\mathrm{cm}^{-1}\right) 1648 ;{ }^{1} \mathrm{H}-\mathrm{NMR}\left(\mathrm{CDCl}_{3}, 200 \mathrm{MHz}\right) \delta 1.05(\mathrm{H}-9,9)$ ), 2.04(H-8), 2.35(H-5), 2.37(H-7), 6.37(H3), 7.08-7.67(Ar-H); MS (EI) m/z (relative intensity) $331\left(\mathrm{M}^{+}, 100\right)$, 333( $\left.\mathrm{M}^{+}+2,100\right)$; HRMS (EI) Calcd for $\mathrm{C}_{17} \mathrm{H}_{18} \mathrm{NOBr} 331.0572$, Found 331.0576.

1-(3-Bromophenyl)-2,6,6-trimethyl-4,5,6,7-tetrahydroindol-4-one (2d). mp 134-135 ${ }^{\circ} \mathrm{C}$; IR $\left(\mathrm{CHCl}_{3}\right)$ $v_{\max }\left(\mathrm{cm}^{-1}\right) 1649 ;{ }^{1} \mathrm{H}-\mathrm{NMR}\left(\mathrm{CDCl}_{3}, 200 \mathrm{MHz}\right) \delta 1.07\left(\mathrm{H}-9,99^{\prime}\right), 2.05(\mathrm{H}-8), 2.35(\mathrm{H}-5), 2.39(\mathrm{H}-7), 6.36(\mathrm{H}-$ 3), 7.09-7.48(Ar-H); MS (EI) m/z (relative intensity) 331 ( $\left.\mathrm{M}^{+}, 100\right)$, 333( $\left.\mathrm{M}^{+}+2,100\right)$; HRMS (EI) Calcd for $\mathrm{C}_{17} \mathrm{H}_{18} \mathrm{NOBr} 331.0572$, Found 331.0574.

1-(3-Chlorophenyl)-2,6,6-trimethyl-4,5,6,7-tetrahydroindol-4-one (2e). mp 129-131 ${ }^{\circ} \mathrm{C}$; $\mathrm{IR}\left(\mathrm{CHCl}_{3}\right)$ $v_{\max }\left(\mathrm{cm}^{-1}\right) 1649 ;{ }^{1} \mathrm{H}-\mathrm{NMR}\left(\mathrm{CDCl}_{3}, 200 \mathrm{MHz}\right) \delta 1.07\left(\mathrm{H}-9,9^{\prime}\right)$, 2.05(H-8), 2.36(H-5), 2.39(H-7), 6.36(H-3),7.14-7.65(Ar-H); MS (EI) m/z (relative intensity) $287\left(\mathrm{M}^{+}, 100\right), 289\left(\mathrm{M}^{+}+2,33.5\right)$; HRMS (EI) Calcd for $\mathrm{C}_{17} \mathrm{H}_{18} \mathrm{NOCl} 287.1077$, Found 287.1079

1-(R-Phenyl)-2,6,6-trimethyl-4,5,6,7-tetrahydroindol-4-one oximes (3a-g). Oximes 3a-g have been prepared following a reported procedure: $3 \mathbf{b}$, mp $209-210{ }^{\circ} \mathrm{C}$ (lit. ${ }^{7} 208-210{ }^{\circ} \mathrm{C}$ ); $3 \mathbf{e}, \mathrm{mp} 175-177{ }^{\circ} \mathrm{C}$ (lit. ${ }^{7} 175-178{ }^{\circ} \mathrm{C}$ ); 3f, mp 220-222 ${ }^{\circ} \mathrm{C}$ (lit. ${ }^{7} 220-223{ }^{\circ} \mathrm{C}$ ); 3 g, mp 213-215 ${ }^{\circ} \mathrm{C}$ (lit. ${ }^{7} 212-213{ }^{\circ} \mathrm{C}$ ). The spectral data where in agreement with the reported data. ${ }^{7}$ Spectral data for the unknown mixture (syn/anti) tetrahydroindol-4-one oximes $\mathbf{3 a} \mathbf{3} \mathbf{3 c}$ and $\mathbf{3 d}$ are described below.

1-(4-Iodophenyl)-2,6,6-trimethyl-4,5,6,7-tetrahydroindol-4-one oximes (syn/anti) (3a). mp 210-212 ${ }^{\circ} \mathrm{C}$, IR $\left(\mathrm{CHCl}_{3}\right) v_{\max }\left(\mathrm{cm}^{-1}\right) 3589 ;{ }^{1} \mathrm{H}-\mathrm{NMR}\left(\mathrm{CDCl}_{3}, 200 \mathrm{MHz}\right) \delta$ 0.96-0.92(H-9,9'), 2.05(H-8), 2.582.25(H-5), 2.37(H-7), 6.82-6.23(H-3), 6.9-7.87(Ar-H); MS (EI) m/z (relative intensity) 394 ( $\mathrm{M}^{+}$, 100),; HRMS (EI) Calcd for $\mathrm{C}_{17} \mathrm{H}_{19} \mathrm{~N}_{2} \mathrm{OI} 394.0542$, Found 394.0554

1-(4-Chlorophenyl)-2,6,6-trimethyl-4,5,6,7-tetrahydroindol-4-one oximes (syn/anti) (3c). mp 185-187 ${ }^{\circ} \mathrm{C}$, IR $\left(\mathrm{CHCl}_{3}\right) v_{\max }\left(\mathrm{cm}^{-1}\right) 3588 ;{ }^{1} \mathrm{H}-\mathrm{NMR}\left(\mathrm{CDCl}_{3}, 200 \mathrm{MHz}\right) \delta$ 0.93-0.90(H-9,9'), 2.06(H-8), 2.58-2.27(H-5), 2.28(H-7), 6.82-6.25(H-3), 7.11-7.48(Ar-H); MS (EI) m/z (relative intensity) $302\left(\mathrm{M}^{+}, 100\right), 304\left(\mathrm{M}^{+}+2\right.$, 33.8); HRMS (EI) Calcd for $\mathrm{C}_{17} \mathrm{H}_{19} \mathrm{~N}_{2} \mathrm{OCl}$ 302.1186, Found 302.1190. 
1-(3-Bromophenyl)-2,6,6-trimethyl-4,5,6,7-tetrahydroindol-4-one oximes (syn/anti) (3d). mp 174$175{ }^{\circ} \mathrm{C}$, IR $\left(\mathrm{CHCl}_{3}\right) v_{\max }\left(\mathrm{cm}^{-1}\right) 3251 ;{ }^{1} \mathrm{H}-\mathrm{NMR}\left(\mathrm{CDCl}_{3}, 200 \mathrm{MHz}\right) \delta 1.03-1.04\left(\mathrm{H}-9,99^{\prime}\right), 2.07(\mathrm{H}-$ 8), 2.61-2.29(H-5), 2.36(H-7), 6.82-6.33(H-3), 7.12-7.62(Ar-H); MS (EI) m/z (relative intensity) $346\left(\mathrm{M}^{+}, 100\right), 348\left(\mathrm{M}^{+}+2,100\right)$; HRMS (EI) Calcd for $\mathrm{C}_{17} \mathrm{H}_{19} \mathrm{~N}_{2} \mathrm{OBr} 346.0681$, Found 346.0684 6H-1-(R-Phenyl)-2,7,7-trimethyl-4,5,7,8-tetrahydropyrrolo[3,2-c]azepin-4-ones (4a-g)

The azepinones 4a-g have been prepared following a reported procedure: $\mathbf{4 b}, \mathrm{mp} 247-249{ }^{\circ} \mathrm{C}$ (lit. ${ }^{7} 247-248{ }^{\circ} \mathrm{C}$ ); 4e, mp 170-171 ${ }^{\circ} \mathrm{C}$ (lit. ${ }^{7} 169-170{ }^{\circ} \mathrm{C}$ ); $\mathbf{4 f}$, mp 248-250 ${ }^{\circ} \mathrm{C}$ (lit. ${ }^{7} 250-251{ }^{\circ} \mathrm{C}$ ); 4g, mp 229-231 ${ }^{\circ} \mathrm{C}$ (lit. ${ }^{7} 230-231{ }^{\circ} \mathrm{C}$ ); The spectral data were in agreement with the reported data. ${ }^{7}$ Spectral data for the unknown azepinones $\mathbf{4 a}, \mathbf{4 c}$, and $\mathbf{4 d}$ are described below.

6H-1-(4-Iodophenyl)-2,7,7-trimethyl-4,5,7,8-tetrahydropyrrolo[3,2-c]azepin-4-one (4a). mp 264-265 ${ }^{\circ} \mathrm{C}$; IR $\left(\mathrm{CHCl}_{3}\right) v_{\max }\left(\mathrm{cm}^{-1}\right)$ 1631; ${ }^{1} \mathrm{H}-\mathrm{NMR}\left(\mathrm{CDCl}_{3}, 200 \mathrm{MHz}\right) \delta 0.96$ (H-10-10'), 1.96 (H-9), 2.31 (H-8), 2.98 (H-6), 6.30 (exchangeable with $\left.\mathrm{D}_{2} \mathrm{O}, \mathrm{N}-\mathrm{H}\right), 6.46$ (H-3), 6.91-7.85 (Ar-H); MS (EI) $\mathrm{m} / \mathrm{z}$ (relative intensity) $394\left(\mathrm{M}^{+}, 100\right)$; HRMS (EI) Calcd for $\mathrm{C}_{17} \mathrm{H}_{19} \mathrm{~N}_{2} \mathrm{OI}$ 394.0542, Found 394.0550 .

6H-1-(4-Chlorophenyl)-2,7,7-trimethyl-4,5,7,8-tetrahydropyrrolo[3,2-c]azepin-4-one (4c). mp 247$249{ }^{\circ} \mathrm{C}$; IR $\left(\mathrm{CHCl}_{3}\right) v_{\max }\left(\mathrm{cm}^{-1}\right) 1635 ;{ }^{1} \mathrm{H}-\mathrm{NMR}\left(\mathrm{CDCl}_{3}, 200 \mathrm{MHz}\right) \delta 0.96(\mathrm{H}-10-10$ '), 1.96 (H-9), 2.33 (H-8), 3.0 (H-6), 6.02 (exchangeable with $\left.\mathrm{D}_{2} \mathrm{O}, \mathrm{N}-\mathrm{H}\right), 6.46$ (H-3), 7.09-7.51 (Ar-H); MS (EI) $\mathrm{m} / \mathrm{z}$ (relative intensity) $302\left(\mathrm{M}^{+}, 100\right), 304\left(\mathrm{M}^{+}+2\right.$, 34); HRMS (EI) Calcd for $\mathrm{C}_{17} \mathrm{H}_{19} \mathrm{~N}_{2} \mathrm{OCl}$ 302.1186, Found 302.1194

6H-1-(3-Bromophenyl)-2,7,7-trimethyl-4,5,7,8-tetrahydropyrrolo[3,2-c]azepin-4-one (4d). mp 178$180{ }^{\circ} \mathrm{C}$; IR $\left(\mathrm{CHCl}_{3}\right) v_{\max }\left(\mathrm{cm}^{-1}\right)$ 1630; ${ }^{1} \mathrm{H}-\mathrm{NMR}\left(\mathrm{CDCl}_{3}, 200 \mathrm{MHz}\right) \delta 0.97(\mathrm{H}-10-10$ '), $1.97(\mathrm{H}-$ 9), 2.32 (H-8), 3.02 (H-6), 6.22 (exchangeable with $\left.\mathrm{D}_{2} \mathrm{O}, \mathrm{N}-\mathrm{H}\right), 6.46(\mathrm{H}-3), 7.11-7.64(\mathrm{Ar}-\mathrm{H})$; MS (EI) $\mathrm{m} / \mathrm{z}$ (relative intensity) $346\left(\mathrm{M}^{+}, 100\right), 348\left(\mathrm{M}^{+}+2,100\right)$; HRMS (EI) Calcd for $\mathrm{C}_{17} \mathrm{H}_{19} \mathrm{~N}_{2} \mathrm{OBr}$ 346.0681, Found 346.0681

6H-1-(R-Phenyl)-2,7,7-trimethyl-4,5,7,8-tetrahydropyrrolo[3,2-c]azepin-4-thiones(4a'-g'). Compounds 4a'-g' have been prepared following a reported procedure: $\mathbf{4 b}$ ', mp $268-270{ }^{\circ} \mathrm{C}$ (lit. ${ }^{5}$ 267-269 ${ }^{\circ} \mathrm{C}$ ); $\mathbf{4 c ^ { \prime }}$, mp 284-286 ${ }^{\circ} \mathrm{C}$ (lit. ${ }^{5} 283-285{ }^{\circ} \mathrm{C}$ ); $\mathbf{4 f}$, mp 271-273 ${ }^{\circ} \mathrm{C}$ (lit. ${ }^{5} 272-274{ }^{\circ} \mathrm{C}$ ); $\mathbf{4 g}$ ', mp 281-283 ${ }^{\circ} \mathrm{C}$ (lit. ${ }^{5} 280-282{ }^{\circ} \mathrm{C}$ ). The spectral data where in agreement with the reported data. ${ }^{5}$ Spectral data for the unknown tetrahydroindol-4-thiones 4a', 4d' and 4e' are described below 6H-1-(4-Iodophenyl)-2,7,7-trimethyl-4,5,7,8-tetrahydropyrrolo[3,2-c]azepin-4-thione (4a'). mp 250-255 ${ }^{\circ} \mathrm{C}$ : IR $\left(\mathrm{CHCl}_{3}\right) v_{\max }\left(\mathrm{cm}^{-1}\right) 1179 ;{ }^{1} \mathrm{H}-\mathrm{NMR}\left(\mathrm{CDCl}_{3}, 200 \mathrm{MHz}\right) \delta 0.95(\mathrm{H}-10,10$ '), 1.94 (H-9), 2.26 (H-8), 3.09 (H-6), 6.65 (H-3), 6.89-7.84 (Ar-H), 8.91 (exchangeable with $\mathrm{D}_{2} \mathrm{O}$, $\mathrm{N}-\mathrm{H}$ ); MS (EI) $\mathrm{m} / \mathrm{z}$ (relative intensity) $410\left(\mathrm{M}^{+}, 100\right)$; HRMS (EI) Calcd for $\mathrm{C}_{17} \mathrm{H}_{19} \mathrm{~N}_{2} \mathrm{IS}$ 410.0314, Found 410.0319.

6H-1-(3-Bromophenyl)-2,7,7-trimethyl-4,5,7,8-tetrahydropyrrolo[3,2-c]azepin-4-thione (4d'). mp 234-235 ${ }^{\circ} \mathrm{C}$ : IR $\left(\mathrm{CHCl}_{3}\right) v_{\max }\left(\mathrm{cm}^{-1}\right)$ 1174; ${ }^{1} \mathrm{H}-\mathrm{NMR}\left(\mathrm{CDCl}_{3}, 200 \mathrm{MHz}\right) \delta 0.99(\mathrm{H}-10,10$ '), 1.97 (H-9), 2.33 (H-8), 3.16 (H-6), 6.62 (H-3), 7.11-7.45 (Ar-H), 9.18 (exchangeable with $\mathrm{D}_{2} \mathrm{O}$, $\mathrm{N}-\mathrm{H})$; MS (EI) m/z (relative intensity) $362\left(\mathrm{M}^{+}, 100\right), 364\left(\mathrm{M}^{+}+2,100\right)$; HRMS (EI) Calcd for $\mathrm{C}_{17} \mathrm{H}_{19} \mathrm{~N}_{2} \mathrm{BrS}$ 362.0452, Found 362.0458. 
6H-1-(3-Chlorophenyl)-2,7,7-trimethyl-4,5,7,8-tetrahydropyrrolo[3,2-c]azepin-4-thione (4e'). mp 201-203 ${ }^{\circ} \mathrm{C}$ : IR $\left(\mathrm{CHCl}_{3}\right) v_{\max }\left(\mathrm{cm}^{-1}\right)$ 1174; ${ }^{1} \mathrm{H}-\mathrm{NMR}\left(\mathrm{CDCl}_{3}, 200 \mathrm{MHz}\right) \delta 0.99$ (H-10, 10'), 1.97 (H-9), 2.31 (H-8), 3.16 (H-6), 6.65 (H-3), 7.11-7.48 (Ar-H), 8.71 (exchangeable with $\mathrm{D}_{2} \mathrm{O}, \mathrm{N}-\mathrm{H}$ ); MS (EI) $\mathrm{m} / \mathrm{z}$ (relative intensity) $318\left(\mathrm{M}^{+}, 100\right), 320\left(\mathrm{M}^{+}+2,38\right)$; HRMS (EI) Calcd for $\mathrm{C}_{17} \mathrm{H}_{19} \mathrm{~N}_{2} \mathrm{ClS}$ 318.0957, Found 318.0965.

6H-1-(R-Phenyl)-2,7,7-trimethyl-4-methylsulfanyl-4,5,7,8-tetrahydropyrrolo[3,2-c]azepines (4a'-g'). Compounds 4a'-g' have been prepared following a reported procedure. The spectral data where in agreement with the reported data. ${ }^{5}$ Compounds $\mathbf{4 a}$ ', $\mathbf{4} \mathbf{b}^{\prime}, \mathbf{4} \mathbf{c}^{\prime}, \mathbf{4} \mathbf{f}^{\prime}$ and $\mathbf{4 g}$ ' are yellow amorphous solid $^{5}$ and they were used without further purification. Spectral data for the unknown methylsulfanylazepines $\mathbf{4 d "}$ and $\mathbf{4} \mathbf{e}$ ” are described below

1-(3-Bromophenyl)-2,7,7-trimethyl-4-methylsulfanyl-4,5,7,8-tetrahydropyrrolo[3,2-c]azepine (4d'). mp 133-135 ${ }^{\circ} \mathrm{C}:{ }^{1} \mathrm{H}-\mathrm{NMR}\left(\mathrm{CDCl}_{3}, 200 \mathrm{MHz}\right) \delta 0.95$ (H-11, 11'), 1.97 (H-9), $2.26(\mathrm{H}-8), 2.43(\mathrm{H}-$ 10), 3.51 (H-6), 6.29 (H-3), 7.11-7.63 (Ar-H); MS (EI) m/z (relative intensity) $376\left(\mathrm{M}^{+}, 100\right)$, $378\left(\mathrm{M}^{+}+2\right.$, 100); HRMS (EI) Calcd for $\mathrm{C}_{18} \mathrm{H}_{21} \mathrm{~N}_{2} \mathrm{BrS}$ 376.0609, Found 376.0618.

1-(3-Chlorophenyl)-2,7,7-trimethyl-4-methylsulfanyl-4,5,7,8-tetrahydropyrrolo[3,2-c]azepine (4e'). mp 118-120 ${ }^{\circ} \mathrm{C}:{ }^{1} \mathrm{H}-\mathrm{NMR}\left(\mathrm{CDCl}_{3}, 200 \mathrm{MHz}\right) \delta 1.04$ (H-11, 11'), 1.98 (H-9), 2.31 (H-8), 2.36 (H-10), 3.57 (H-6), 6.34 (H-3), 7.08-7.49 (Ar-H); MS (EI) m/z (relative intensity) $332\left(\mathrm{M}^{+}, 100\right), 334$ $\left(\mathrm{M}^{+}+2\right.$, 38); HRMS (EI) Calcd for $\mathrm{C}_{18} \mathrm{H}_{21} \mathrm{~N}_{2}$ ClS 332.1114, Found 332.1120.

3-(R-Phenyl)-2,5,5-trimethyl-9a-methylsulfanyl-9-phenoxy-4,5,6,8,9,9a-hexahydro-3H-azeto[1,2a]pyrrolo[3,2-c]azepin-8-one (1a-g). Compounds 1a-g have been prepared following a reported procedure. The spectral data where in agreement with the reported data. ${ }^{5,7} \mathbf{1 a}, \mathrm{mp} 195-196{ }^{\circ} \mathrm{C}$ (lit. ${ }^{3}$ 194-195 ${ }^{\circ} \mathrm{C}$ ); 1b, mp $183-186{ }^{\circ} \mathrm{C}$ (lit. ${ }^{5}$ yellow oil); 1c, mp $178-180{ }^{\circ} \mathrm{C}$ (lit. ${ }^{5} 177-179{ }^{\circ} \mathrm{C}$ ); $\mathbf{1 f}$, mp 171-173 ${ }^{\circ} \mathrm{C}$ (lit. ${ }^{5} 170-171{ }^{\circ} \mathrm{C}$ ); $\mathbf{1 g}$, mp 184-185 ${ }^{\circ} \mathrm{C}$ (lit. ${ }^{5} 181-183{ }^{\circ} \mathrm{C}$ ). Spectral data for the unknown azeto-pyrrolo azepinone $\mathbf{1 d}$ and $\mathbf{1 e}$ are described below

3-(3-Bromophenyl)-2,5,5-trimethyl-9a-methylsulfanyl-9-phenoxy-4,5,6,8,9,9a-hexahydro$3 \mathrm{H}$-azeto[1,2-a]pyrrolo[3,2-c]azepin-8-one (1d). mp 159-162 ${ }^{\circ} \mathrm{C}$ : IR $\left(\mathrm{CHCl}_{3}\right) v_{\max }\left(\mathrm{cm}^{-1}\right)$ 1755; ${ }^{1} \mathrm{H}-\mathrm{NMR}\left(\mathrm{CDCl}_{3}, 200 \mathrm{MHz}\right) \delta 0.91$ (H-12), 0.95 (H-11), 1.9 (H-10), 2.1 (H-4b), $2.19(\mathrm{H}-12)$, 2.69 (H-4a), 3.05 (H-6b), 3.66 (H-6a), 5.5 (H-1), 5.8 (H-9), 7.01- 7.60 (Ar-H); MS (EI) m/z (relative intensity) $510\left(\mathrm{M}^{+}\right.$, 9), $512\left(\mathrm{M}^{+}+2,9\right)$; HRMS (EI) Calcd for $\mathrm{C}_{26} \mathrm{H}_{27} \mathrm{~N}_{2} \mathrm{O}_{2} \mathrm{BrS} 510.0977$, Found 510.0988

3-(3-Chlorophenyl)-2,5,5-trimethyl-9a-methylsulfanyl-9-phenoxy-4,5,6,8,9,9a-hexahydro-3Hazeto[1,2-a]pyrrolo[3,2-c]azepin-8-one (1e). mp 162-164 ${ }^{\circ} \mathrm{C}$; IR $\left(\mathrm{CHCl}_{3}\right) v_{\max }\left(\mathrm{cm}^{-1}\right) 1756$; ${ }^{1} \mathrm{H}$ NMR ( $\left.\mathrm{CDCl}_{3}, 200 \mathrm{MHz}\right) \delta 0.91(\mathrm{H}-12), 0.94$ (H-11), 1.89 (H-10), 2.17 (H-4b), $2.19(\mathrm{H}-12)$, 2.61 (H-4a), 3.04 (H-6b), 3.74 (H-6a), 5.51 (H-1), 5.8 (H-9), 7.08- 7.43 (Ar-H); MS (EI) m/z (relative intensity) $466\left(\mathrm{M}^{+}, 12\right), 468\left(\mathrm{M}^{+}+2,5\right)$; HRMS (EI) Calcd for $\mathrm{C}_{26} \mathrm{H}_{27} \mathrm{~N}_{2} \mathrm{O}_{2} \mathrm{ClS}$ 466.1482, Found 466.1489.

\section{Cytotoxic activity}

Tumoral cell lines were supplied by the National Cancer Institute. The cytotoxicity assays were carried out at 5000 to 7500 cells/ml. as reported by Skehan et al and Monks et al using the 
sulforhodamine $\mathbf{B}$ (SRB) protein assay to estimate cell growth. ${ }^{8,9}$ Compounds were dissolved in DMSO which has not effect on the inhibition has shown by the control. The \% inhibition of the growth described for all compounds were obtained from three different experiments. The percentage growth was evaluated spectrophotometrically in a Bio kinetics reader spectrophotometer. Daunomicyne was used as reference. This compound under the described conditions gave $100 \%$ of inhibition. Each experiment was made two times by triplicate.

\section{Acknowledgments}

We thank DGAPA-UNAM (IN-211601) for financial support. We also thank R. Patiño, H. Rios, A. Peña, N. Zavala, L. Velasco and J. Pérez for technical assistance. Contribution No. 1772 from Instituto de Química, UNAM.

\section{References}

1. Gupta, S. P. Chem. Rev. 1994, 94, 1507.

2. Chacón-García, L.; Martínez, R. Eur. J. Med. Chem. 2002, 37, 261.

3. Martínez, R.; Ávila-Zárraga, J. G.; Duran M. E.; Ramírez, M. T.; Cañas, R. Bioorg. Med. Chem. Lett. 2002, 12, 1675.

4. Proudfoot, J. R. Bioorg. Med. Chem. Lett. 2002, 12, 1647.

5. Martínez, R.; Avila-Zárraga, J. G.; López-López, G.; Nava-Salgado, V. O. Heterocycles 2000, 53, 557.

6. Martínez, R.; Avila-Zárraga, J. G.; Reyes, E. Synth. Commun. 1995, 25,1071.

7. Martínez, R.; López, G.; Avila-Zárraga, J. G.; J. Heterocyclic Chem. 1995, 32, 491.

8. Skehan, P.; Storeng, R.; Scudiero, D.; Monks, A.; McMahon, J.; Vistica, D.; Warren, J. T.; Bokesch, H.; Kenney, S.; Boyd, M. R. J. Nat. Cancer Inst. 1990, 82, 1107.

9. Monks, A.; Scudiero, D.; Skehan, P.; Shoemaker, R.; Paull, K.; Vistica, D.; Hose, C.; Langley, J.; Cronise, P.; Vaigro-Wolff, A.; Gray-Goodrich, M.; Campbell, H.; Mayo, J.; Boyd, M. J. Nat. Cancer Inst. 1991, 83, 757 